\title{
La evaluación de la ciencia en España. La necesaria compatibilización de los estándares formales y materiales de evaluación científica*
}

\author{
Leonardo Álvarez Álvarez ${ }^{\star *}$ \\ Profesor de Derecho Constitucional en la Universidad de Oviedo \\ Secretario de la Revista Electrónica de Historia Constitucional \\ Ignacio Fernández Sarasola ${ }^{\star \star \star}$ \\ Profesor de Derecho Constitucional en la Universidad de Oviedo \\ Director de la Revista Electrónica de Historia Constitucional
}

\section{SUMARIO}

1. La creación de la ANECA como pretensión de centralizar y objetivar la evaluación de la calidad científica / 2. El procedimiento de acreditación ante la ANECA y la CNEAI / La formalización del proceso de evaluación de la calidad científica / 3. Hacia una necesaria redefinición material de la evaluación de la calidad científica: la sentencia del Tribunal Supremo, de 12 de junio de 2018 / 4. Quid custodiat ipso custodes? La arbitrariedad de las agencias evaluadoras de la calidad científica de las revistas / 5. Retomando el control: una alternativa puramente científica / 6 . Conclusiones

\section{RESUMEN}

Este trabajo pretende someter a enjuiciamiento los criterios empleados por los organismos de evaluación de la investigación científica en España en el ámbito de las ciencias sociales. Estos criterios han transcurrido hacia una formalización que descuida el contenido material de lo publicado. Sobre ello ha advertido una reciente sentencia del Tribunal Supremo, de 12 de junio de 2018. El trabajo pretende formular una propuesta para compatibilizar, en el ámbito de las revistas jurídicas, la necesaria existencia de criterios formales de evaluación con los materiales requeridos por las exigencias de la ciencia.

\section{PALABRAS CLAVE}

Criterios de evaluación, ranking de revistas científicas, formalización de la investigación, indexación.

\footnotetext{
* Artículo de investigación Número Especial de Aniversario. Recibido el 12 de marzo de 2020 y aceptado para su publicación el 24 de junio de 2020.

** Profesor Titular en la Universidad de Oviedo, España. / leonardo@uniovi.es / orcid. org/0000-0002-3388-2586

*** Profesor Titular en la Universidad de Oviedo, España. / sarasola@uniovi.es / orcid. org/ 0000-0002-2638-4108
} 


\section{La creación de la ANECA como pretensión de centralizar y objetivar la evaluación de la calidad científica}

La Ley Orgánica de Universidades 6/2001, de 21 de diciembre, previó en su art. 32 la formación de una Agencia Nacional de Evaluación de la Calidad y Acreditación (ANECA). Dicha Agencia fue definitivamente constituida por el Acuerdo del Consejo de Ministros, de 19 de julio de 2002. La creación de la ANECA, en 2002, supuso un punto de inflexión en la evaluación de la calidad de la producción científica del profesorado universitario. Hasta entonces, había regido un modelo evaluativo caracterizado principalmente por dos notas: a) la descentralización y b) la subjetividad de la valoración de la producción científica. Con anterioridad a 2002, la valoración de la producción científica se encontraba fundamentalmente descentralizada en los distintos tribunales y comisiones de evaluación que se constituían ad hoc en el seno de cada universidad (en ejercicio de la autonomía universitaria reconocida constitucionalmente) para la provisión de los diferentes puestos universitarios. Además, la valoración de la producción científica descansaba, en buena medida, en la subjetividad de cada uno los miembros que integraban aquellos tribunales y comisiones. Ello, en principio, condujo a una pluralidad y a una indefinición de los criterios de evaluación. Sin embargo, a pesar de que ello pudiera ser así, lo cierto es que el escaso número de revistas y editoriales que existía en aquel momento relativizaba, en cierto modo, esa subjetividad de las evaluaciones de la producción científica.

Tampoco suponía, en realidad, una excepción a este modelo de evaluación basado principalmente en la descentralización y en la subjetividad de la existencia de la Comisión Nacional Evaluadora de la Actividad Investigadora (CNEAI), que venía funcionando desde 1989 para evaluar la calidad de la labor investigadora desarrollada cada seis años por el profesorado universitario.

Representó, es cierto, la pretensión de crear un organismo centralizado de la evaluación; ${ }^{1} \sin$ embargo, la labor de la CNEAI no logró la subjetividad de la evaluación ni su carácter descentralizado debido, sobre todo, a tres motivos.

En primer lugar, porque su labor evaluadora no se apoyaba en unos estándares objetivos de calidad convenientemente organizados y estructurados. En segundo

\footnotetext{
${ }^{1}$ Acerca de la evolución del sistema de evaluación del profesorado y sus características, véase: Aguado Cudolà, Vicenç, "La selección de los cuerpos docentes universitarios", Revista de Educación y Derecho, núm. 10, 2014, pp. 3-8.
} 
lugar, porque la valoración positiva de cada periodo investigador de seis años poseía tan solo efectos económicos. ${ }^{2}$ En tercer lugar —el más relevante-, porque solo podían solicitar su evaluación los integrantes de los cuerpos docentes universitarios (profesores titulares y catedráticos de universidad) que ya habían sido evaluados previa y positivamente por los tribunales y comisiones constituidas en cada universidad para la provisión de sus puestos. Seguían siendo, por tanto, estos sobre los que seguía descansando la labor principal de la evaluación de la calidad científica de las publicaciones.

La ansiada objetivación y centralización de dicha evaluación sí pudo conseguirla la creación de la ANECA en 2002; primero, porque la ANECA centralizó de manera eficaz el enjuiciamiento de la producción científica. Este órgano se encargaría de emitir una valoración positiva como requisito necesario para poder concurrir a la mayoría de los puestos docentes universitarios. Ningún tribunal ni comisión podían proceder a la provisión de puestos sin que los candidatos presentaran la correspondiente acreditación positiva por parte de la ANECA. En segundo lugar, la ANECA consiguió también, hasta cierto punto, acabar con la subjetividad de la valoración de la producción científica con la paulatina creación de estándares objetivos organizados y estructurados de calidad que acabarían extendiéndose también a las evaluaciones realizadas por la CNEAI.

\section{El procedimiento de acreditación ante la ANECA y la CNEAI. La formalización del proceso de evaluación de la calidad científica}

Que la centralización y, sobre todo, la objetivación de los criterios llamados a enjuiciar la calidad científica eran necesarios queda fuera de toda duda; sin embargo, ello ha conducido a una formalización tal de dichos estándares que ha acabado por renunciar prácticamente a cualquier valoración material de su contenido. ${ }^{3}$ Así ha venido a reconocerlo la sentencia del Tribunal Supremo, de 12 de junio de 2018, de la que se hablará más adelante. De la formalización de los estándares evaluativos da cumplida cuenta el procedimiento de evaluación

\footnotetext{
2 Véase: Alfageme González, M. Begoña y Caballero Rodríguez, Katia, "Evaluación y profesorado en la universidad española", Revista Educatio Siglo XXI, vol. 28, núm. 1, p. 278.

${ }^{3}$ Véase: Fernández Sarasola, Ignacio, "Algunas reflexiones en torno a la evaluación del profesorado universitario de ciencias jurídicas", Revista General de Derecho Constitucional, núm. 21, 2015, pp. 1-18.
} 
ante la ANECA y la ANECA, ${ }^{4}$ un procedimiento basado en una inversión de la carga de la prueba de la calidad científica de las publicaciones, gravada, en ocasiones, por ciertas presunciones.

En efecto, de manera paradójica, los organismos que desempeñan la función de evaluar las publicaciones científicas trasladan al solicitante la labor de acreditar, según los casos, su calidad. Tal inversión de la carga de la prueba se ve en ocasiones grabada, además, por determinadas presunciones formales y materiales. En lo que a las primeras se refiere, el sistema de evaluación parte de que la existencia de más de un autor (al margen de cuál sea su prestigio) o un relativamente escaso número de páginas (independientemente de los resultados que han sido defendidos en ellas) reducen exponencialmente y por sí mismos el valor de la publicación sometida a evaluación. ${ }^{5}$

En otras ocasiones, las presunciones construidas en contra de la calidad científica se vinculan con prejuicios materiales ligados al objeto de lo publicado: las lecciones de manuales, los comentarios jurisprudenciales, los comentarios críticos de monografías o las voces de enciclopedias, al margen de si son el resultado de una verdadera investigación científica, no son, en principio, tenidas en cuenta para las evaluaciones positivas de la calidad científica. A partir de estas presunciones, el solicitante de evaluación, bien por sí mismo o bien necesariamente coadyuvado por un tercero (un editor), debe ser quien asuma la carga de probar la calidad de lo publicado, algo que depende del formato en el que se recojan las publicaciones.

En el caso de los libros y los capítulos de libros, que han desempeñado tradicionalmente un relevante protagonismo en el ámbito de las ciencias sociales, los organismos de evaluación parten de su inferior valor natural. Es directamente el evaluado el que debe acreditar su calidad, a partir del número de citas recibidas y la relevancia académica de su trabajo. El mayor o menor prestigio de la editorial $y$, en general, el consustancial inferior valor de lo publicado en este tipo de formatos posiblemente haya tenido algo que ver con el

\footnotetext{
4 Un detenido examen del procedimiento de evaluación puede verse en Moretón Sanz, M ${ }^{\underline{a}}$ Fernanda, "Selección, evaluación y acreditación del profesorado ante el espacio europeo de educación superior", Revista de Derecho UNED, núm. 5, 2009, pp. 357-360.

${ }^{5}$ García Rubio, M. Paz, "La evaluación de la investigación en las humanidades y las ciencias sociales, con especial referencia al caso del derecho", Revista El Cronista del Estado Social y Democrático de Derecho, núm. 32, 2012, pp. 42-58.
} 
hecho de que las editoriales, incluso algunas públicas, hayan ido transitando hacia el modelo de cofinanciación por parte de quien aspira a publicar en ellas. En el caso de las revistas, el procedimiento ante la ANECA y ante la CNEAI parte, en principio, del superior valor académico de tales formatos, modulado, cierto es, por los rankings elaborados por diferentes organismos. Estos rankings han respondido a la proliferación de revistas en el ámbito académico, muchas de ellas con formato electrónico, que han sorteado las dificultades procedimentales y pecuniarias a las que se venían enfrentando las tradicionales revistas. El reconocimiento de un superior valor científico de los artículos de revistas ha acabado por aplicar al ámbito de las ciencias sociales un modelo de evaluación que se había difundido en el plano de las ciencias naturales y en otras disciplinas sociales, como la economía.

Es en el caso de las publicaciones de revistas, el solicitante de evaluación debe ser coadyuvado necesariamente por un tercero para acreditar la calidad de lo publicado. El procedimiento de evaluación ante la ANECA y ante la CNEAI requiere que los editores de revistas científicas soliciten a diferentes organismos, ya sean públicos o privados, los correspondientes sellos de calidad.

Son, en realidad, estos editores quienes habrán de soportar indirectamente la carta de la prueba de avalar la calidad científica de la obra sometida a evaluación ante aquellos organismos. ${ }^{6}$ Más adelante se analizará de qué manera se ha regulado este procedimiento para obtener las correspondientes acreditaciones de calidad de las distintas revistas.

\section{Hacia una necesaria redefinición material de la evaluación de la calidad científica: la sentencia del Tribunal Supremo, de 12 de junio de 2018}

El procedimiento actualmente vigente de evaluación de la calidad científica, con el loable fin de centralizar y de objetivar los estándares evaluativos, ha conducido en la práctica a una casi total formalización, desvinculándose del contenido académico de lo publicado. Así ha venido a reconocerlo la sentencia del Tribunal Supremo (2524/2018), de 12 de junio de 2018. Esta sentencia resuelve un recurso de casación interpuesto frente a diferentes resoluciones que

\footnotetext{
${ }_{6}^{6}$ Una crítica de este sistema de evaluación puede verse en Bastida Freijedo, Francisco, "La nueva carrera académica: visión senior (con vista cansada)", Revista General de Derecho Constitucional, núm. 21, 2015 (consultada: 12 de marzo de 2020). Disponible en: https://www.iustel.com/v2/revistas/detalle_revista.asp?id=3\&numero=21.
} 
desestimaron los recursos interpuestos contra una resolución de la CNEAI que evaluaba negativamente un periodo de investigador de seis años.

El Tribunal Supremo deja claro que:

no es suficiente para decidir si las aportaciones sometidas a la evaluación de la Comisión Nacional merecen o no un juicio técnico favorable o positivo la consideración de la publicación en la que han aparecido (...) Las investigaciones, las aportaciones presentadas por los interesados, no pueden dejar de examinarse sólo por el hecho de que no se publicaran en las revistas o medios incluidos en los índices o listados identificados en la resolución de 26 de noviembre de 2014 ... Dependerá de su contenido la evaluación que merezcan.

En definitiva, a través de dicha sentencia el Tribunal Supremo ha impuesto, sobre todo, un criterio material para la evaluación de la calidad investigadora. Desde luego, mucho más propio con los estándares característicos de la labor científica. Ésta no debe se evaluada exclusivamente con arreglo a los criterios formales que han edificado la esencia del procedimiento de evaluación ante los organismos a los que se les ha atribuido dicha función. De esta exigencia se deriva la necesidad de reelaborar los criterios con los cuales los diferentes organismos públicos y privados han procedido a definir los estándares de calidad de las revistas científicas. A la descripción crítica de cuáles han sido estos durante los últimos y a la defensa de una de las maneras posibles de compatibilizar los criterios formales con los necesariamente materiales propios del pensamiento científico se dedican las siguientes páginas.

\section{Quid custodiat ipso custodes? La arbitrariedad de las agencias evaluadoras de la calidad científica de las revistas}

Las revistas jurídicas se han incorporado a una dinámica establecida para las publicaciones de ciencias experimentales, y lo han hecho de forma acrítica, sin asumir las profundas diferencias que existen entre uno y otro campo del conocimiento y, por tanto, también en el modo de difundirlo. ${ }^{7}$

\footnotetext{
${ }^{7}$ Para un análisis más detallado de cuanto aquí se señala, véase Fernández Sarasola, Ignacio, "Los índices de impacto de las revistas jurídicas. Fraude y negocio", Revista El Cronista del Estado Social y Democrático de Derecho, núm. 50, 2015, pp. 58-67.
} 
Los criterios que se emplean para evaluar si una revista tiene calidad científica son decididos, asignados e impuestos por diferentes actores. En ocasiones cuentan con el soporte de instituciones públicas, como sucede con Latindex, Redalyc o Recyt; en otros casos, se trata de empresas privadas como Thomson Reuters o Scopus. Sin duda, el caso más grave lo representan estas últimas, por la evidente circunstancia de que privilegian sus propios productos e imponen a los demás editores ajustarse a los parámetros que ellos han decidido, arbitrariamente, para sus publicaciones. ${ }^{8}$ La situación es tan absurda como si una empresa automovilística impusiese a todas las demás la mecánica y electrónica de sus propios vehículos, al punto de que, de no ajustarse a sus estándares de ingeniería, se considerase que los demás automóviles no deberían ni siquiera circular.

Pero incluso en el caso de agencias respaldadas por instituciones públicas el procedimiento empleado para evaluar las revistas jurídicas resulta igualmente espurio. El problema de base reside en que la decisión de cuándo una revista jurídica tiene o no calidad se ha dejado en manos de biblioteconomistas que desconocen totalmente el contenido de las publicaciones. Han buscado, por tanto, establecer unos parámetros de calidad que sean válidos para toda publicación —cualquiera que sea la ciencia de la que verse-, algo sólo posible si esa calidad se fía exclusivamente a aspectos formales, desentendiéndose de lo que debiera primar: el contenido.

Tomemos como referencia Latindex, por tratarse de la base de datos que establece los criterios de calidad más estandarizados en el ámbito académico. El Catálogo 2.0 de Latindex atiende 38 variables, de las cuales 8 están pensadas específicamente para las revistas digitales. A pesar de que estos 38 factores están englobados en 5 categorías distintas (características básicas, de presentación, de gestión y política editorial, de contenido y de revistas en línea), un examen más detenido pone en evidencia que, en realidad, todos esos criterios

\footnotetext{
${ }^{8}$ Kein, Daniel B. y Chiang, Eric, "The social science citation index: a black box - with an ideological bias", Econ Journal Watch, vol. 1, núm. 1, 2004, 6|. 134-165. Otras limitaciones funcionales de esta base de datos se relatan en Castro Spila, Javier; Barrenechea, Julieta e Ibarra, Andoni, "Indicadores de actividad científica en ciencias sociales y humanidades: hacia un modelo integral basado en patrones disciplinarios de investigación", La evaluación de la actividad científica en ciencias sociales y humanidades, Servicio Editorial de la Universidad del País Vasco, 2007, pp. 9-11.
} 
responden tan solo a dos variables: la gestión de la revista y sus aspectos formales. Incluso las denominadas "características del contenido" nada tienen que ver con el contenido, sino con el continente.

Con la única excepción de la originalidad del trabajo —algo que, por otra parte, es poco concreto: ¿es original una revisión de un trabajo previo ya publicado?-, todas las demás exigencias se centran en aspectos puramente formales, como la cantidad de artículos publicados al año o la presencia de resumen y palabras clave, unos factores volumétricos, idiomáticos —resumen en dos idiomas... ¿y si el segundo es una lengua muerta o minoritaria? ¿Cuál es su utilidad?_ y que, en definitiva, no sirven en absoluto para determinar la calidad del contenido. Es como si en el ámbito académico evaluásemos los trabajos de nuestros alumnos no ya por su solvencia jurídica, sino exclusivamente por el modo en que están presentados: número de páginas, que vayan grapados y que estén en una tipografía determinada.

Tan poco riguroso método - acientífico, en realidad- para evaluar calidad científica pretende, en algunas bases de indexación, complementarse computando la cantidad de citas que reciben los trabajos publicados en esas revistas. Procedimiento que emplea, por ejemplo, Web of Science o, en España, los índices IN-RECJ O IN-RECS, elaborados por un grupo de biblioteconomistas de la Universidad de Granada. ${ }^{9}$

Tal procedimiento tiene una apariencia de objetividad: cuanto más citada sea una revista, se entiende que más valor científico tiene. Pero obviamente enseguida se aprecia que se trata de una falacia. En primer lugar, porque, por ejemplo, Thomson Reuters privilegia las citas que se efectúen a "revistas de calidad", entre las que se encuentran —cómo no- las que él mismo edita. De este modo se entra en un espurio bucle: la empresa dice qué es de calidad, y si no citas esas publicaciones, tú no entrarás en el gremio; pero al citarlas estás

\footnotetext{
9 Especialmente activo en España, este grupo ha defendido sus arbitrarios criterios de clasificación de las revistas, entre otras publicaciones, en DELGADO LÓPEZ-CÓZAR, EMILIO; RUIZ-PÉREZ, RAFAEL; JIMÉNEZ-CONTRERAS, EVARISTO, LA EDICIÓN DE REVISTAS CIENTÍFICAS. DIRECTRICES, CRITERIOS Y MODELOS DE EVALUACIÓN, UNIVERSIDAD DE GRANADA, 2006, P. 66. Por cierto, que los apellidos de los autores están falseados por los propios autores, al introducir en ellos guiones, lo que contraría lo dispuesto por la Dirección General de Registros y Notariado, que señala que la modificación de los apellidos no queda al arbitrio de los particulares, debiendo someterse a las reglas españolas de Derecho Civil.
} 
engordando artificialmente el prestigio de las revistas de la empresa que elabora el índice. Todo un fraude.

Pero no es el único. Por ejemplo, los índices IN-RECJ e IN-RECS - cuyo acceso web, por cierto, estaba plagado de pop-ups de publicidad-se han elaborado sin tener en cuenta, por ejemplo, las citas que una revista recibe en libros o en publicaciones internacionales. El resultado es que una revista cuyos trabajos se citen a menudo en el ámbito doméstico puede llegar a ser mejor valorada que otra citada a menudo a nivel internacional. Por si fuera poco, no se discrimina entre el tipo de revistas, lo que entraña un error metodológico que invalida totalmente la ponderación: una revista generalista siempre tiene más citas que otra especializada en un campo muy concreto, aun cuando esta última pueda ser todo un referente internacional en ese campo. Es como si obligásemos a un deporte minoritario, como el lanzamiento de martillo, a competir en subvenciones con el fútbol: es obvio que, siendo más los federados en este último deporte, también acabarán recibiendo más remuneración. Por si fuera poco, las citas en libros no se contemplan, demostrando, como ya se ha dicho, que en el actual sistema de evaluación resulta más rentable publicar un pequeño artículo en una revista fraudulentamente considerada "de calidad" que redactar un libro completo tras años de estudio.

En definitiva, es obvio que nos hallamos ante una pura formalización y cuantificación de la calidad. Estando las agencias de indexación en manos de biblioteconomistas, la forma se ha impuesto sobre el contenido. Ello no ha supuesto en absoluto una mejora en la calidad de las publicaciones: la necesidad de publicar cada vez más (al peso) para obtener sexenios y acreditaciones ha dado lugar, en realidad, a que el nivel de exigencia mengüe. Así las cosas, lo primero que habría que evaluar es precisamente a esos grupos de evaluación, cuya opacidad y arbitrariedad lastran la calidad de las publicaciones.

\section{Retomando el control: una alternativa puramente científica}

El desenfoque con el que actualmente se atiende la calidad de las revistas sólo puede superarse de una forma certera: siendo los propios conocedores de la materia, es decir, los juristas, los que determinen qué revistas de su gremio tienen o no calidad. Los criterios formales no deben ser los que imperen, del 
mismo modo que un libro puede ser bueno o malo al margen del tipo de papel en que esté impreso, que tenga contraportada o que se edite en formato de lujo. Retomar el control significa, pues, una autoevaluación, es decir, que quienes podemos ponderar el contenido seamos los que aquilatemos las revistas a las que acudimos para ampliar nuestros conocimientos y para dar a conocer los resultados de nuestras pesquisas. Por desgracia, se trata de una política excepcional que, por ejemplo, en España sólo aplicó Revistas Españolas de Ciencias Sociales y Humanidades (RESH), utilizando como uno de sus criterios de calidad la "opinión de los expertos", recabada a partir de entrevistas a once mil profesores universitarios que tuviesen al menos reconocido un sexenio de investigación. El sistema, sin embargo, no resultó eficaz: por una parte, porque la última encuesta ya está muy obsoleta (2009); por otra, porque lo que se pidió a los encuestados es que seleccionaran las tres revistas más relevantes de su especialidad. El número tan escaso de revistas que se podían seleccionar, unido al hecho de que no se discriminara su temática, daba lugar a que las generalistas copasen los primeros puestos. Dicho de otro modo: no existía proporcionalidad alguna.

Para limar estos problemas, lo que aquí se propone es promover encuestas elaboradas por un equipo de expertos integrado por profesores de reconocido prestigio en la disciplina jurídica de que se trate. De existir una asociación nacional de juristas de esa disciplina, podría ser la que promoviese y organizase la confección de la encuesta. Como alternativa, podría ser una agencia estatal (como en España la ANECA) la que organizase el proceso.

Resulta imprescindible que en él se pida al encuestado que elija o indique las revistas (en un número limitado) que considera más prestigiosas, pero dentro de bloques temáticos. Es precisamente en la decisión de cuáles habrán de ser estos en donde el rigor académico debe quedar garantizado. Cada disciplina jurídica propondrá sus particulares campos temáticos que obviamente dependen de ella, pero siempre habrá una categoría que esté presente: las revistas generalistas, es decir, aquellas en las que tiene cabida cualquier tema de esa disciplina. Éstas deben suponer siempre un segmento aparte de los restantes, ya que de lo contrario serían siempre las más valoradas, según ya se ha señalado. 
Así, a modo de ejemplo, en el ámbito del derecho constitucional en España sería posible tomar como base estos temas: ${ }^{10}$

\section{Generalistas}

2. Derecho constitucional comparado

3. Historia constitucional

4. Teoría del Estado y de la Constitución

5. Democracia, participación y partidos políticos

6. Estado social

7. Comunidades autónomas y entes locales

8. Parlamento y derecho parlamentario

9. Jurisprudencia constitucional

10. Derechos fundamentales: revistas generalistas

11. Derechos fundamentales: revistas específicas

Por supuesto, esta clasificación de revistas pretende ser sólo una propuesta a modo de bases, que habría que meditar y sería susceptible de matices, ampliando, reduciendo o ramificando, en su caso, los campos referidos. Un añadido que daría más valor a la encuesta sería incluir un listado de todas las revistas de la disciplina publicadas en el país, a fin de evitar descuidos a la hora de cumplimentar el formulario.

Elaborada la encuesta, el siguiente paso sería determinar sus destinatarios. Si lo que va a evaluarse son revistas científicas, parece que el criterio más razonable es circunscribir las respuestas a las provenientes del profesorado universitario. El criterio utilizado por RESH de exigir un sexenio de investigación puede ser también una ponderación adecuada: un sexenio no es un objetivo inalcanzable, sino proporcionado, y además garantiza que quien responda a la encuesta ya ha demostrado su valía investigadora.

\footnotetext{
10 Una encuesta aproximada a la que aquí propongo fue elaborada para las revistas de derecho civil por el grupo dirigido por el profesor Pablo Salvador: Salvador Coderch, Pablo; Azagra Malo, Albert y Gómez Ligüerre, Carlos Ignacio, "Criterios de evaluación de la actividad investigadora en derecho civil, derecho privado y análisis del derecho", Indret: Revista para el Análisis del Derecho, Núm. 3, 2008, pp. 43-49.
} 
Las encuestas deberían ser recabadas por el organismo responsable, que daría publicidad a los resultados a través de una página web en la que se mostrase el orden de preferencias dentro de cada bloque temático. Ahora bien, todo este proceso sería fútil si las universidades y agencias estatales no asumieran la plena validez de los resultados a efectos de concesión de becas y proyectos de investigación, reconocimiento de méritos investigadores y acreditaciones de profesorado. Por ese motivo, sería conveniente que todo el proceso contase con el respaldo de las universidades y organismos dependientes del Ministerio de Educación.

Sin duda, se podrán objetar inconvenientes, porque nada es perfecto. Pero al menos con este sistema seremos los propios conocedores de la materia los que decidamos qué tiene o no calidad. Si nos equivocamos, que sea por méritos propios y no por imposiciones externas que respondan a intereses empresariales o a puros formalismos.

\section{Conclusiones}

La creación de agencias estatales de evaluación, como la ANECA o la CNEAI, supuso una necesaria centralización de la competencia para evaluar el conocimiento científico. Sin embargo, la creación de la ANECA, en 2002, implicó la paulatina introducción de criterios formales para la valoración de la calidad de las publicaciones, extendiéndose, sobre todo, para las revistas, que son las que poseen mayor relevancia en la evaluación. Tales criterios formales, que también han sido asumidos por la CNEAI, han conducido a una evaluación que prescinde prácticamente del contenido de lo publicado, tal como ha reconocido la sentencia del Tribunal Supremo, de 12 de junio de 2018.

Por tanto, es necesario redefinir los criterios de evaluación de las publicaciones realizadas en revistas científicas que sean capaces de compatibilizar criterios formales y materiales. Una fórmula para hacerlo posible es elaborar un ranking de revistas no sólo basado en los primeros, como sucede en la actualidad. Para ello se propone un modelo en el que se consulte a los expertos, a los propios juristas, una iniciativa que debe de ser promovida, ya sea por las asociaciones nacionales que los aúna o por las agencias nacionales de evaluación, como la ANECA o la CNEAI. Además, es necesario que la valoración de las revistas por 
parte de los expertos se haga según campos temáticos, sin perjuicio que se reserve uno para las revistas generalistas.

\section{BIBLIOGRAFÍA CITADA:}

Aguado Cudolà, Vicenç, "La selección de los cuerpos docentes universitarios", Revista de Educación y Derecho, núm. 10, 2014, pp. 1-21.

Alfageme González, M. Begoña y Caballero Rodríguez, Katia, "Evaluación y profesorado en la universidad española", Revista Educatio Siglo XXI, vol. 28, núm. 1, pp. 271-293.

Bastida Freijedo, Francisco, "La nueva carrera académica: visión senior (con vista cansada), Revista General de Derecho Constitucional, núm. 21, $2015 . \quad$ Disponible en: https://www.iustel.com/v2/revistas/detalle revista.asp?id=3\&numero=21

Castro Spila, Javier; Barrenechea, Julieta y Ibarra, Andoni, "Indicadores de actividad científica en ciencias sociales y humanidades: hacia un modelo integral basado en patrones disciplinarios de investigación", La evaluación de la actividad científica en ciencias sociales y humanidades, Servicio Editorial de la Universidad del País Vasco, 2007, pp. 73-103.

Delgado López-Cózar, Emilio; Ruiz-Pérez, Rafael y Jiménez-Contreras, Evaristo, La edición de revistas científicas. Directrices, criterios y modelos de evaluación, Universidad de Granada, 2006.

Fernández Sarasola, Ignacio, "Algunas reflexiones en torno a la evaluación del profesorado universitario de ciencias jurídicas", Revista General de Derecho Constitucional, núm. 21, 2015, pp. 1-18.

Fernández Sarasola, Ignacio, "Los índices de impacto de las revistas jurídicas. Fraude y negocio", Revista El Cronista del Estado Social y Democrático de Derecho, núm. 50, 2015, pp. 58-67.

García Rubio, M. Paz, "La evaluación de la investigación en las humanidades y las ciencias sociales, con especial referencia al caso del derecho", Revista El Cronista del Estado Social y Democrático de Derecho, núm. 32, 2012, pp. 42-58.

kein, Daniel B. y Chiang, Eric, "The social science citation index: a black box with an ideological bias", Econ Journal Watch, vol. 1, núm. 1, 2004, pp. 134-165.

Moretón Sanz, Ma. Fernanda, "Selección, evaluación y acreditación del profesorado ante el espacio europeo de educación superior", Revista de Derecho UNED, núm. 5, 2009, pp. 319-371.

Salvador Coderch, Pablo; Azagra Malo, Albert y Gómez Ligüerre, Carlos Ignacio, "Criterios de evaluación de la actividad investigadora en derecho civil, derecho privado y análisis del derecho", Indret: Revista para el Análisis del Derecho, núm. 3, 2008, pp. 1-63. 